\title{
Analysis of Full-Period and Non-full-Period Sampling of Vibration Signal for Engine Rotors
}

\author{
Jun PI \& Xiangji BU \\ Sino-European Institute of Aviation Engineering, Civil Aviation University of China, Tianjin, China.
}

ABSTRACT: In this paper, some main software implementations concerning full-period sampling and nonfull-period sampling are cited. Re-sampling technique based on cubic spline interpolation and harmonic analysis algorithm are representative methods of respectively full-period sampling and non-full-period sampling. The advantages and limitations of the two methods are compared by means of MATLAB simulation and experiment.

Analysis of results finds that the re-sampling technique can completely restore the periodical signals and effectively avoid spectral leakage and fence effect, but it needs a large amount of operations. When synchronous error is considerable the compute of period can be inaccurate. Harmonic analysis algorithm of non-full-period sampling can avoid spectral leakage caused by synchronization error and has a great tolerance for deviation. Moreover, it can make the harmonic analysis surpass the Shannon theorem. However, it can only analyze integer multiple frequency signal.

KEYWORD: Vibration signal; full-period sampling; non-full-period sampling; spectral leakage

\section{GENERAL INSTRUCTIONS}

The vibration signal of aero-engine rotor is one of the most important signals reflecting the working status. So monitoring and analyzing rotor vibration signals are important methods to monitor and predict engine failure. Time domain analysis and spectrum analysis of vibration signals are commonly used analysis methods. Time domain analysis can only roughly determine whether there is a fault. But spectrum analysis can analyze the dynamic characteristics and extract fault features of the object. Thus, spectrum analysis is commonly used in work condition monitoring and fault diagnosis.

\section{FULL-PERIOD SAMPLING}

\subsection{Introduction of full-period sample method}

Full period sampling is a method he length of each intercepted sampling signal are equal to an integer multiple of the vibration signal period.

Software-based sampling methods have been widely studied, V Staudt and his colleagues

Support by central university basic research

Civil Aviation University of China special funding:

ZXH2012H003 estimated signal period by multiplying the signal and the window function, then transform them by fast Fourier[4], CI Byrnes and colleagues obtained signal period through filtering signals by the filter array[2]. WEN Hongju and his colleagues implemented fullperiod re-sampling through using software to synchronously collect vibration signals and speed signals[5]. The first two methods cannot guarantee the accuracy of the period. WEN's method can only capture full period signals which do not contain synchronic phase information of vibration signal.

Currently more feasible method is Re-sampling Technique raised by ZHANG Ren. This method requires a key phase signal and vibration signals sampled simultaneously, and then use the software to analyze the collected data, according to the key phase frequency gained by calculation we can apply full period truncation and interpolation to obtain full-period sampled data.

\subsection{Re-sampling Technology}

To get synchronous full-period re-sampled vibration signal, we must first find the starting and ending point of each period. While the key phase signal contains a start and end point, we can find a phase marker to mark them, and then using cubic spline interpolation method to sample them. The main 
process is calculating the period via the key phase signal. The algorithm is[7]:

1) Calculate the peak-to-peak value $\left(V_{P P}\right)$ and average value $\left(\mathrm{V}_{\text {ave }}\right)$

2) Search sequentially the data of key phase signal, if less than $\left(\mathrm{V}_{\mathrm{PP}}+0.5 \mathrm{~V}_{\mathrm{ave}}\right)$ set it to 0 , go on until find the first data greater than $\left(\mathrm{V}_{\mathrm{PP}}\right.$ $+0.5 \mathrm{~V}_{\mathrm{ave}}$ ), set it 1 . Then set the data behind to 0 until there is a data less than $\left(\mathrm{V}_{\mathrm{PP}}+0.4\right.$ $\mathrm{V}_{\text {ave }}$ ).

3) Repeat step 2 to find the position of every key phase pulse.

From the interval and number of sampling points between two adjacent keys phase signals, the sampling period and rotational speed can be calculated.

Apply cubic spline interpolation method to vibration signals, and then re-sampling the signal.

Theorem of cubic spline interpolation: in the interval $[a, b]$, there are $n+1$ points $\left(x_{j}, y_{j}\right), j=0$, 1,Ln. Assuming that $a=x_{0}<x_{1}<L<x_{n}=b$, if the function satisfies:

- It is a cubic polynomial on each subinterval $\left[x_{j-1}, y_{j}\right], \quad j=1,2, L n$.

- $\mathbf{S}\left(x_{j}\right)=y_{j}, j=1,2, L n$., which means that the function's curve pass by all the points.

- In interval $[a, b]$ it exists first and second order deviation, to ensure smoothness of the curve.

It is called Cubic Spline Interpolation function. Applying this theorem to the original time sampling data, we can better approximate the original vibration signal, and then we can re-sample the signal. Steps are as follows:

1) According to processed key phase signals, we can get the number of samples $\mathrm{N}$ between two adjacent peaks (one period). Since the speed of rotor is increasing, $\mathrm{N}$ will gradually reduce in the horizontal direction.

2) As we set the sampling frequency 64 points per period, so $n=N / 64$ is the sampling interval of one period.

3) Because key phase signal and timing sampling signal are collected simultaneously, their horizontal axils is one to one correspondence, so we can sample with the step of $n$ from the vibration signals processed by cubic spline interpolation algorithm which begins from the initial position of coordinate. So that we can obtain the full-period sampled data.

\section{NON-FULL-PERIOD SAMPLING}

\subsection{Introduction of non-full-period sampling}

In actual situation it's impossible to achieve strict synchronization. It always exists synchronization error which can produce large errors in the calculation. For this reason, many scholars have proposed a variety of harmonic spectrum analysis method to inhibit or reduce the synchronization deviation. For example: windowing technique and windowing interpolation techniques[1], quasisynchronous sampling method[3]. These methods have more or less measurement errors in principle. The harmonic analysis algorithm, put forward by ZHANG Jianqiu in 1995, applies non-full-period sampling method in harmonic analysis of periodic signals, and deduced correct and clear conclusion. This algorithm eliminate the spectral leak errors regardless whether the actual sampling synchronous or not. It breaks the restriction of Shannon sampling theorem.

\subsection{Harmonic Analysis Algorithm of Non-Full- Period Sampled signal}

Harmonic analysis algorithm[6]: assuming $f(t)$ is a periodic signal on $\left[t_{0}, t_{0}+T-T_{0}\right]$, we apply nonfull-period sampling on it with sample frequency $T_{S}$ $=(T-\Delta T) / N$, where $\Delta$ is the synchronization error, and $-0.5<\Delta<0.5, \mathrm{~T}$ is signal period. We sample $N+2$ points within the sampling period $T_{S}$. Which are:

$$
f\left(t_{0}\right), f\left(t_{0}+T_{s}\right), \ldots, f\left(t_{0}+K T_{s}\right), \ldots, f\left(t_{0}+N T_{s}\right), f\left[t_{0}+(N+1) T_{s}\right]
$$

with $K=0,1, \ldots, N+1$. Calculation definitions are given as follows:

$$
\begin{gathered}
\frac{Q}{P}=\frac{f\left(t_{0}\right)+f\left(t_{0}+T_{s}\right)-f\left[t_{0}+(N+1) T_{s}\right]-f\left(t_{0}+N T_{s}\right)}{f\left[t_{0}+(N+1) T_{s}\right]+f\left(t_{0}+T_{s}\right)-f\left(t_{0}+N T_{s}\right)-f\left(t_{0}\right)} \\
(1-\Delta)=\frac{P N}{P N+Q}
\end{gathered}
$$

$\frac{Q_{m a}}{P_{m a}}=\frac{f\left(t_{0}\right)+f\left(t_{0}+T_{s}\right) \cos [2 \pi m(1-\Delta) / N]-f\left[t_{0}+(N+1) T_{s}\right]}{f\left[t_{0}+(N+1) T_{s}\right] \cos [2 \pi m(1-\Delta)(N+1) / N]+f\left(t_{0}+T_{s}\right)} \rightarrow$

$\leftarrow \frac{\cos [2 \pi m(1-\Delta)(N+1) / N]-f\left(t_{0}+N T_{s}\right) \cos [2 \pi m(1-\Delta)]}{\cos [2 \pi m(1-\Delta) / N]-f\left(t_{0}+N T_{s}\right) \cos [2 \pi m(1-\Delta)]-f\left(t_{0}\right)}$

$a_{m}=\frac{2}{N+\frac{Q_{m a}}{P_{m a}}}\left\{\begin{array}{l}\frac{f\left(t_{0}\right)+f\left(t_{0}+N T_{s}\right) \cos [2 \pi m(1-\Delta)]}{2}\left(1+\frac{Q_{m a}}{P_{m a}}\right)+ \\ \sum_{K=1}^{N-1} f\left(t_{0}+K T_{s}\right) \cos \frac{2 \pi m(1-\Delta)}{N} K\end{array}\right\}$

$\frac{Q_{m b}}{P_{m b}}=\frac{f\left(t_{0}+T_{s}\right) \sin [2 \pi m(1-\Delta) / N]-f\left[t_{0}+(N+1) T_{s}\right]}{f\left[t_{0}+(N+1) T_{s}\right] \sin [2 \pi m(1-\Delta)(N+1) / N]+f\left(t_{0}+T_{s}\right)} \rightarrow$

$\leftarrow \frac{\sin [2 \pi m(1-\Delta)(N+1) / N]-f\left(t_{0}+N T_{s}\right) \sin [2 \pi m(1-\Delta)]}{\sin [2 \pi m(1-\Delta) / N]-f\left(t_{0}+N T_{s}\right) \sin [2 \pi m(1-\Delta)]}$

$$
b_{m}=\frac{2}{N+\frac{Q_{m b}}{P_{m b}}}\left\{\begin{array}{l}
\frac{f\left(t_{0}+N T_{s}\right) \sin [2 \pi m(1-\Delta)]}{2}\left(1+\frac{Q_{m b}}{P_{m b}}\right)+ \\
\sum_{K=1}^{N-1} f\left(t_{0}+K T_{s}\right) \sin \frac{2 \pi m(1-\Delta)}{N} K
\end{array}\right\}
$$


Where $m=1,2, \ldots M$ ( $M$ is the highest harmonics order), by this algorithm the harmonic's amplitude and phase of each order can be obtained:

$$
\left\{\begin{array}{l}
A_{m}=\overline{a_{m}^{2}+b_{m}^{2}} \\
\varphi_{m}=\arctan \left(a_{m} / b_{m}\right)
\end{array}\right.
$$

This algorithm has been demonstrated there is no measurement error in theory.

\section{VERIFICATION}

In this paper we use software simulation and experimental data to verify and compare the feasibility and the accuracy of the harmonic analysis and cubic spline interpolation re-sampling method.

\subsection{Simulation verification}

Software simulations will implemented by MATLAB. The simulation signal is superimposed sinusoidal signal:

$$
f(t)=\sum_{m=1}^{4} m \times \sin (m k t)
$$

The key phase signal can be simulated by pulse signal with to be determined and great enough amplitude. In this simulation we set $\mathrm{N}=64$, synchronization error $\triangle$ change from $1 \%$ to $10 \%$. We re-sample the signals in different synchronization error using respectively the fullperiod sampling method and non-full-period sampling method. Table 1 and table 2 are results. Am and $\varphi_{\mathrm{m}}$ represent respectively measured amplitude and phase. Error represents the deviation of measured amplitude compares with the amplitude of input signal.

\begin{tabular}{|c|c|c|c|c|c|c|c|}
\hline \multicolumn{2}{|c|}{ Harmonic order } & \multicolumn{3}{|c|}{1 st } & \multicolumn{3}{|c|}{ 2nd } \\
\hline & $\triangle$ & $\mathrm{Am}$ & error & $\varphi_{\mathrm{m}} /\left(^{\circ}\right)$ & $\mathrm{Am}$ & error & $\varphi_{\mathrm{m}} /\left(^{\circ}\right)$ \\
\hline \multirow{6}{*}{$\begin{array}{l}\overrightarrow{0} \\
\stackrel{0}{0} \\
\stackrel{0}{1} \\
\stackrel{\vec{I}}{\Xi}\end{array}$} & 0.01 & 0.9949 & 0.0051 & -90.2781 & 1.9822 & 0.0089 & -90.4325 \\
\hline & 0.02 & 0.9887 & 0.0113 & -90.4993 & 1.9500 & 0.0250 & -90.8429 \\
\hline & 0.04 & 0.9542 & 0.0458 & -91.3640 & 1.9250 & 0.0375 & -91.5872 \\
\hline & 0.06 & 0.9012 & 0.0988 & -91.9824 & 1.7680 & 0.1160 & -92.3640 \\
\hline & 0.08 & 0.8794 & 0.1206 & -89.4512 & 1.7536 & 0.1232 & -88.7451 \\
\hline & 0.10 & 0.7220 & 0.2780 & -88.6938 & 1.3454 & 0.3273 & -86.3896 \\
\hline \multirow{6}{*}{ 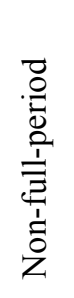 } & 0.01 & 0.9931 & 0.0069 & -90.4762 & 1.979 & 0.0105 & -90.531 \\
\hline & 0.02 & 0.9946 & 0.0054 & -90.4276 & 1.9776 & 0.0112 & -90.8429 \\
\hline & 0.04 & 0.9902 & 0.0098 & -90.5382 & 1.9654 & 0.0173 & -91.0154 \\
\hline & 0.06 & 0.9723 & 0.0277 & -90.9364 & 1.9316 & 0.0342 & -91.6357 \\
\hline & 0.08 & 0.9085 & 0.0915 & -91.0437 & 1.7682 & 0.1159 & -89.4185 \\
\hline & 0.10 & 1.0089 & 1.0089 & -89.7165 & 2.0266 & 1.0133 & -88.8672 \\
\hline
\end{tabular}

Table 1. Simulation result of the 1st and 2nd order harmonic.

\begin{tabular}{|c|c|c|c|c|c|c|c|}
\hline \multicolumn{2}{|c|}{ Harmonic order } & \multicolumn{3}{|c|}{$3 \mathrm{rd}$} & \multicolumn{3}{|c|}{ 4th } \\
\hline & $\triangle$ & $\mathrm{Am}$ & error & $\varphi_{\mathrm{m}} /\left(^{\circ}\right)$ & $\mathrm{Am}$ & error & $\left.\varphi_{\mathrm{m}} / /^{\circ}\right)$ \\
\hline \multirow{6}{*}{ 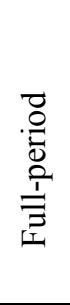 } & 0.01 & 2.9589 & 0.0137 & -91.2746 & 3.9212 & 0.0197 & -91.679 \\
\hline & 0.02 & 2.8848 & 0.0384 & -92.8345 & 3.7952 & 0.0512 & -93.3977 \\
\hline & 0.04 & 2.7162 & 0.0946 & -94.7421 & -0.1676 & 1.0419 & -88.4538 \\
\hline & 0.06 & 2.5785 & 0.1405 & -89.3698 & -0.2776 & 1.0694 & -85.5512 \\
\hline & 0.08 & 2.3805 & 0.2065 & -86.4859 & -1.1528 & 1.2882 & -86.2467 \\
\hline & 0.10 & -0.7323 & 1.2441 & -82.3674 & -1.4368 & 1.3592 & -80.1596 \\
\hline \multirow{6}{*}{ 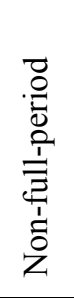 } & 0.01 & 2.9544 & 0.0152 & -91.3355 & 3.9196 & 0.0201 & -91.7579 \\
\hline & 0.02 & 2.9472 & 0.0176 & -92.2813 & 3.9208 & 0.0198 & -92.9852 \\
\hline & 0.04 & 2.9514 & 0.0162 & -93.6575 & 3.9048 & 0.0238 & -94.6551 \\
\hline & 0.06 & 2.8452 & 0.0516 & -94.2568 & 3.7036 & 0.0741 & -88.7963 \\
\hline & 0.08 & 3.0951 & 1.0317 & -88.7466 & 4.0896 & 1.0224 & -87.5887 \\
\hline & 0.10 & 3.0807 & 1.0269 & -87.6539 & 4.2756 & 1.0689 & -85.6122 \\
\hline
\end{tabular}

Table 2. Simulation result of the 3rd and 4th order harmonic. 


\subsection{Experiment verification}

The experiment verification is based on the data acquired from the own-build rotor experiment platform in laboratory. The data is sampled by a high performance data acquisition card in which vibration signal and key phase signal are acquired in different channels. A continuous acquired stable signal for a period of time $t$ is set as a reference. This signal mainly concentrated on base frequency and double frequency parts. The experimental data is a section of this signal. By calculation on MATLAB we can obtain respectively the errors calculated by different method in different synchronization error.

Table 3. Errors of different sampling method.

\begin{tabular}{|l|l|l|l|l|l|}
\hline Harmonic order & $\Delta$ & 1st & 2nd & 3rd & 4th \\
\hline \multirow{4}{*}{ Full-period } & 0.01 & 0.0094 & 0.0071 & 0.0827 & 0.1432 \\
\cline { 2 - 6 } & 0.02 & 0.0263 & 0.0269 & 0.1755 & 0.3754 \\
\cline { 2 - 6 } & 0.04 & 0.0951 & 0.0193 & 0.1476 & 0.1120 \\
\cline { 2 - 6 } & 0.06 & 0.2420 & 0.2638 & 0.3845 & 0.3421 \\
\hline \multirow{4}{*}{ Non-full-period } & 0.01 & 0.0133 & 0.0103 & 0.0351 & 0.1075 \\
\cline { 2 - 6 } & 0.02 & 0.0215 & 0.0483 & 0.0874 & 0.1429 \\
\cline { 2 - 6 } & 0.04 & 0.0659 & 0.0989 & 0.0796 & 0.2385 \\
\cline { 2 - 6 } & 0.06 & 0.1827 & 0.2134 & 0.2533 & 0.2472 \\
\hline
\end{tabular}

\subsection{Results analysis}

From the result shown on the tables, we can easily conclude that:

- Both of these two methods can restore the signal when the synchronization error $\triangle$ is small.

- They have very high accuracy when $\triangle<2 \%$, and the full-period sampling is a little superior to harmonic analysis algorithm of non-full-period.

- The errors increase as the synchronization error becomes lager. The re-sampling results are in high accuracy when $\triangle<4 \%$ for full-period but for non-full-period re-sampling the tolerance is $\triangle<$ $8 \%$ (6\% in experimental verification).

- According to the experiment result, the harmonic analysis algorithm of non-full-period can only acquire the integer multiple frequency signals while filtering the non-integer frequency.

\section{CONCLUSIONS}

Analysis of the results finds that both of the resampling technique and harmonic analysis algorithm can completely restore the periodical signals and the accuracy is high when the synchronization error is small. The re-sampling technique can effectively avoid spectral leakage and fence effect, but the interpolation and re-sampling processes need a large amount of operations. When variation of rotating speed is considerable the compute of period can be inaccurate. Harmonic analysis algorithm can avoid spectral leakage error caused by synchronization error and has a relatively great tolerance for deviation. Moreover, it can make the harmonic analysis surpass Shannon theorem. However, it can only analyze integer multiple frequency signal. Some improvement methods, proposed by same scholars, endow this method with the ability to analyze arbitrary harmonics but the filter will cause loss of data.

Since the synchronous error of aero-engine rotor is not great, and the signal has often integer multiple frequency, these two methods can restore the signal in high accuracy. But considering the amount of computation, hardware devices performance, tolerance for synchronization deviation, non-fullperiod sampling algorithm has obvious advantages.

\section{REFERENCES}

[1] Andria. G. Windows and Interpolation Algorithms to Improve Electrical Measurement Accuracy IEEE Trans. IM-38 No.4, (1989).

[2] Byrnes C I, Georgiou T T , Lindquist A . A new approach to spectral estimation: A tunable high-resolution spectral estimator. IEEE Trans. Signal Processing, 48:3189 3205.

[3] Dai Zhongxian. Methods for Improving the Accuracies of Quasi-synchronous Sampling Harmonic Analysis. Chinese Journal of Scientific Instrument. 1992, 13(4): 350-357. Chinese.

[4] StaudtV. Effects of window-function explained by signal's typical to power electronics IEEE 8th Int. Conf .Harmonics Quality Power(ICHQP), vol. II, Oct. 1998:952 - 957. Athens, Greece.

[5] WenHongju, Sun Tao, cte. The Arithmetic and the Implement of the Order $\mathrm{T}$ racking of Rotating Machinery Based on PCI Data Acquisition Card. Mechanical and electronic. 2006, 03: 16-18. Chinese.

[6] Zhang Jian-qiu, Tao Ran, Shen, Yi. The application of Non-Integer-Period Sampling Method in Spectrum Analysis of Periodic Signals. Chinese Journal of Scientific Instrument. 1995, 16(1). Chinese.

[7] Zhang Ren, Yang Shixi, cte. A full period sampling method based on the application of re-sampling technology. Mechanical \& electrical magazine. 2004. 21(3): 57-59. Chinese. 